\title{
Efeito da auto liberação miofascial na força muscular em atletas de futebol feminino: ensaio clínico randomizado
}

\section{Effect of self-myofascial release on muscle strength in female soccer athletes: randomized clinical trial}

\author{
Samuel Miranda Souza' ${ }^{1}$ (1) \\ José Fernando Pereira Costa Neto ${ }^{2}$ (1) \\ Jorge Eduardo Tavares Santos ${ }^{3}$
}

${ }^{1}$ Autor para correspondência. Universidade Católica do Salvador (Salvador). Bahia, Brasil. samuel.souza@ucsal.edu.br ${ }^{2}$ Universidade Católica do Salvador (Salvador). Bahia, Brasil. fnandoneto@hotmail.com ${ }^{3}$ Faculdade de Tecnologia e Ciências (Salvador). Bahia, Brasil.jorgetavaresfisio@yahoo.com.br

\begin{abstract}
RESUMO | INTRODUÇÃO: A variável força muscular (FM) na prática esportiva, sobretudo no futebol, é de extrema importância em atividades como chutes, disputas de bola, arrancadas e saltos. Além disso, permite identificar indivíduos que estão em grupos de risco para lesões. Assim, técnicas, como a auto liberação miofascial (ALM), vêm sendo utilizadas para o aprimoramento do desempenho esportivo e para prevenir lesões. OBJETIVO: Verificar o efeito crônico da ALM na FM em atletas de futebol feminino. METODOLOGIA: Este estudo trata-se de um ensaio clínico randomizado. Participaram do estudo 14 atletas de futebol feminino. Inicialmente foram realizadas avaliações no aparelho dinamômetro isocinético dos extensores e flexores de joelho. As participantes foram divididas em dois grupos, um grupo intervenção (GI) e um grupo controle (GC). As participantes do Gl realizaram um aquecimento associado à ALM com um foam roller. Já as participantes do GC, realizaram o mesmo aquecimento, porém sem a associação da ALM. Após 48 horas da última intervenção, foi realizada uma reavaliação no aparelho dinamômetro isocinético. RESULTADOS: Não foram verificados efeitos significativos entre os grupos no pós-teste. Na comparação pré e pós-teste entre os grupos, houve uma piora significativa na variável pico de torque de extensão do membro inferior dominante no grupo que não fez a ALM $(p=0,013)$. CONCLUSÃo: Conclui-se que uma abordagem crônica de ALM não foi capaz de gerar mudanças significativas na FM dos extensores e flexores de joelho. Mais estudos são necessários para apoiar esses achados. Registro ReBEC: RBR-7qnxty.
\end{abstract}

PALAVRAS-CHAVE: Auto liberação miofascial. Fáscia. Força muscular. Futebol. Atletas.

\begin{abstract}
INTRODUCTION: The variable muscle strength (MS) in sports practice, especially in football, is extremely important in activities such as kicks, ball disputes, sprints and jumps. In addition, it allows identifying individuals who are at risk groups for injuries. Thus, techniques, such as selfmyofascial release (SMF), have been used to improve sports performance and to prevent injuries. OBJECTIVE: To verify the chronic effect of SMF on MS in female soccer athletes. METHODOLOGY: This study is a randomized clinical trial. 14 female soccer athletes participated in the study. Initially, evaluations were performed on the isokinetic dynamometer device of knee extensors and flexors. The participants were divided into two groups, an intervention group (IG) and a control group (CG). IG participants performed a warm-up associated with SMF with a foam roller. CG participants, on the other hand, performed the same warm-up, but without the association of SMF. 48 hours after the last intervention, a reassessment was performed on the isokinetic dynamometer device. RESULTS: There were no significant effects between groups in the post-test. In the pre- and post-test comparison between the groups, there was a significant worsening in the peak torque variable of the dominant lower limb extension in the group that did not perform SMF $(p=0.013)$. CONCLUSION: It is concluded that a chronic SMF approach was not able to generate significant changes in the MS of knee extensors and flexors. Further studies are needed to support these findings. ReBEC Registration: RBR-7qnxty.
\end{abstract}

KEYWORDS: Self-myofascial release. Fascia. Strength muscle. Soccer. Athletes. 


\section{Introdução}

A prática do futebol necessita de variadas exigências físicas para um bom desempenho, demandando atributos básicos como a flexibilidade, mobilidade, agilidade $^{1}$ e força muscular (FM) ${ }^{1,2}$. Sabe-se que a variável FM na prática esportiva, sobretudo no futebol, é de extrema importância em atividades como chutes, disputas de bola, arrancadas e saltos ${ }^{2}$. Além disso, permite identificar indivíduos que estão em grupos de risco para lesões ${ }^{3}$. Acredita-se que a capacidade de transmitir FM pode ser influenciada, além de diversos fatores, pela integridade do sistema miofascial (isto é, músculo e fáscia) $)^{4,5}$.

O sistema miofascial exerce importante função na transmissão de forças mecânicas entre músculos, podendo frequentemente apresentar alterações, como perda da sua elasticidade e viscosidade, o que geralmente leva a densificações, que estão associadas a dor, alteração da mecânica muscular, diminuição da flexibilidade e FM ${ }^{5}$. Assim, algumas técnicas, como a auto liberação miofascial (ALM), vêm sendo recentemente utilizadas para o aprimoramento do desempenho esportivo e para prevenir lesões ${ }^{6}$.

A ALM consiste numa técnica de automassagem em que o indivíduo utiliza a sua própria massa corporal para gerar pressão em uma determinada região do corpo com um instrumento específico. A técnica tornou-se amplamente utilizada no meio esportivo como um componente adicional de um aquecimento, ganhando notoriedade no campo do condicionamento físico ${ }^{7}$. No futebol, a ALM pode ser aplicada para impedir restrições na amplitude de movimento de membros inferiores, que contribui na prevenção de lesões ${ }^{6}$.

Estudos recentes mostram efeitos positivos da ALM utilizada como uma estratégia de abordagem em atletas, prés,9,10 e pós-exercício ${ }^{11,12,13}$. De modo geral, a literatura sugere que no pré-exercício a ALM é capaz de melhorar a flexibilidade, sem prejudicar o desempenho muscular durante a prática esportiva, e no pós-exercício, é capaz de melhorar a recuperação muscular ${ }^{14,15}$. Quanto aos efeitos da ALM sobre a $\mathrm{FM}$, a maioria dos estudos não encontrou nenhuma relação de causa e efeito ${ }^{10,16,17}$, porém, todos eles avaliaram os efeitos agudos da técnica. Entende-se por efeitos agudos, os estudos que realizaram um protocolo menor que uma semana ${ }^{15}$. Portanto, o objetivo deste estudo foi verificar o efeito crônico da ALM sobre a FM em atletas de futebol feminino.
Metodologia

Este estudo trata-se de um ensaio clínico randomizado com amostra definida por conveniência. Fizeram parte da pesquisa 14 atletas de futebol feminino, integrantes do Esporte Clube Vitória (ECV), na cidade de Salvador - Bahia, onde foi realizada a coleta de dados. Foram incluídas na pesquisa as atletas de futebol feminino do ECV. Foram excluídas da pesquisa as atletas que estavam em processo de recuperação de lesões nos membros inferiores, que realizaram cirurgia nos últimos oito meses que antecederam o período de coleta e que apresentou alguma condição que as incapacitasse de realizar as práticas. Os instrumentos utilizados para a realização desta pesquisa foram um questionário sociodemográfico e clínico, um aparelho dinamômetro isocinético Biodex e rolos de massagem Foam Roller Brasil ${ }^{\circledR}$ Original - $30 \times 15 \mathrm{~cm}$.

As participantes foram divididas em dois grupos equivalentes aleatoriamente, caracterizando um grupo intervenção e um grupo controle. A aleatorização foi realizada pelo preparador físico do clube, sem a participação dos pesquisadores, e se deu por meio de uma listagem numerada de 1 a 14 das atletas participantes e em seguida um sorteio por meio de um aplicativo de celular responsável por gerar números aleatórios. Primeiramente o sorteio alocou as participantes para o grupo intervenção, composto de sete participantes. As outras sete participantes que restaram, foram alocadas automaticamente para o grupo controle.

Inicialmente foram realizadas avaliações no apareIho dinamômetro isocinético dos extensores e flexores de joelho. Após 48 horas da avaliação isocinética, deu-se início as intervenções, que seguiu um protocolo de duas semanas, sendo realizadas três vezes durante a semana. As participantes do grupo intervenção realizaram um aquecimento de rotina do clube associado à ALM com um foam roller. Já as participantes do grupo controle realizaram o mesmo aquecimento, porém sem a associação da ALM. O protocolo da ALM foi aplicado pelo colaborador do estudo e preparador físico do clube, que não participou das avaliações, e foi realizado nos grupos musculares quadríceps, isquiostibiais e tríceps sural, sendo realizadas três séries de 1 minuto para cada grupo muscular, com 30 segundos de descanso entre as séries. Ao total foram realizadas seis intervenções com intervalo de 48 horas entre cada. Após 48 horas da última intervenção, foi realizada uma reavaliação no aparelho dinamômetro isocinético. Para este estudo, 
a variável analisada do resultado da avaliação, tanto pré-teste, quanto pós-teste, foi o pico de torque de extensão e flexão do joelho a uma velocidade angular de $60 \%$ do membro dominante e não dominante. $O$ protocolo utilizado neste estudo no que se refere ao número de séries, tempo de ALM e tempo de descanso foi baseado na literatura anterior ${ }^{10,11,12,17}$.

Para a análise descritiva da amostra, os dados foram apresentados com média, desvio padrão, frequência absoluta e frequência relativa. Para verificar a existência de resultados significativos pré e pós-intervenção realizou-se o teste t-Student para amostras pareadas, e para comparar os efeitos entre os grupos, realizou-se o teste t-independente. Todas as análises estatísticas foram realizadas através do programa IBM SPSS Statistics 20.0. O nível de significância atribuído foi $p<0,05$.

Este estudo foi aprovado pelo Comitê de Ética e Pesquisa da Universidade Católica do Salvador (CAAE 07858819.1.0000.5628) e registrado no Registro Brasileiro de Ensaios Clínicos (ReBEC: RBR-7qnxty). Os procedimentos foram executados dentro das normas éticas previstas na Resolução de n 466/12 do Conselho Nacional de Saúde em pesquisas envolvendo seres humanos. Todas as participantes assinaram o termo de consentimento livre e esclarecido.

\section{Resultados}

Após análise do questionário sociodemográfico e clínico respondido pelas atletas participantes, nenhuma delas se enquadrou nos critérios de exclusão estabelecidos na metodologia desta pesquisa, totalizando inicialmente uma amostra de 15 participantes. Contudo, uma das atletas da população, não participou do protocolo na fase de intervenção, desta forma, sendo excluída do estudo. Portanto, a amostra deste estudo foi composta de 14 participantes, divididas aleatoriamente em dois grupos, grupo intervenção $(n=7)$ e grupo controle $(n=7)$.

Quanto aos dados descritivos da amostra, o grupo intervenção apresentou médias de idade de 22,3 $\pm 2,3$ anos, peso $64 \pm 10 \mathrm{~kg}$, estatura $1,7 \pm 0,1 \mathrm{~cm}$, IMC 22,4 $\pm 2,4 \mathrm{~kg} / \mathrm{m} 2$ e tempo médio de prática profissional no futebol de $7 \pm 1,4$ anos. Já o grupo controle apresentou média de idade $22,7 \pm 4,3$ anos, $62 \pm 7,6 \mathrm{~kg}$, estatura $1,7 \pm 0,1 \mathrm{~cm}, \mathrm{IMC} 21 \pm 1,5 \mathrm{~kg} / \mathrm{m} 2$ e o tempo médio de prática profissional no futebol de 5,3 \pm 5,8 anos (dados descritos na tabela 1).

Tabela 1. Dados descritivos da amostra (idade, peso, estatura, índice de massa corporal e tempo de atuação profissional) do grupo intervenção e do grupo controle

\begin{tabular}{|c|c|c|}
\hline $\begin{array}{l}\text { Variável } \\
(\mathrm{N}=7)\end{array}$ & $\begin{array}{l}\text { Grupo intervenção } \\
\qquad(N=7)\end{array}$ & Grupo controle \\
\hline & média \pm DV & média \pm DV \\
\hline Idade (anos) & $22,3 \pm 2,3$ & $28,8 \pm 4,3$ \\
\hline Peso (kg) & $64 \pm 10$ & $62 \pm 7,6$ \\
\hline Estatura $(\mathrm{cm})$ & $1,7 \pm 0,1$ & $1,7 \pm 0,1$ \\
\hline IMC (Kg/m2) & $22,4 \pm 2,4$ & $21 \pm 1,5$ \\
\hline $\begin{array}{l}\text { Tempo de atuação } \\
\text { profissional (anos) }\end{array}$ & $7 \pm 1,4$ & $5,3 \pm 5,8$ \\
\hline
\end{tabular}

IMC = Índice de massa corporal. 
Das 14 atletas participantes, $12(85,7 \%)$ eram destras e $02(14,3 \%)$ sinistras. Quanto à posição de jogo, $03(21,4 \%)$ eram goleiras, $02(14,3 \%)$ zagueiras, 01 (7,1\%) lateral e zagueira, $04(28,6 \%)$ meio campistas e $04(28,6 \%)$ atacantes.

Após analisar os resultados pós-teste dos grupos (controle e intervenção), foi possível identificar que o grupo intervenção apresentou maiores médias para todas as variáveis analisadas, quando comparadas ao grupo controle, porém, não apresentou significância estatística ao analisar os valores de P para nenhuma das variáveis. Valores de P: pico de torque de extensão do membro dominante $=0,399$; extensão membro não dominante $=$ 0,681; flexão membro dominante $=0,184$; flexão membro não dominante $=0,588$ (dados descritos no gráfico 1 ).

Gráfico 1. Valores de pico de torque pós-teste dos grupos intervenção e controle

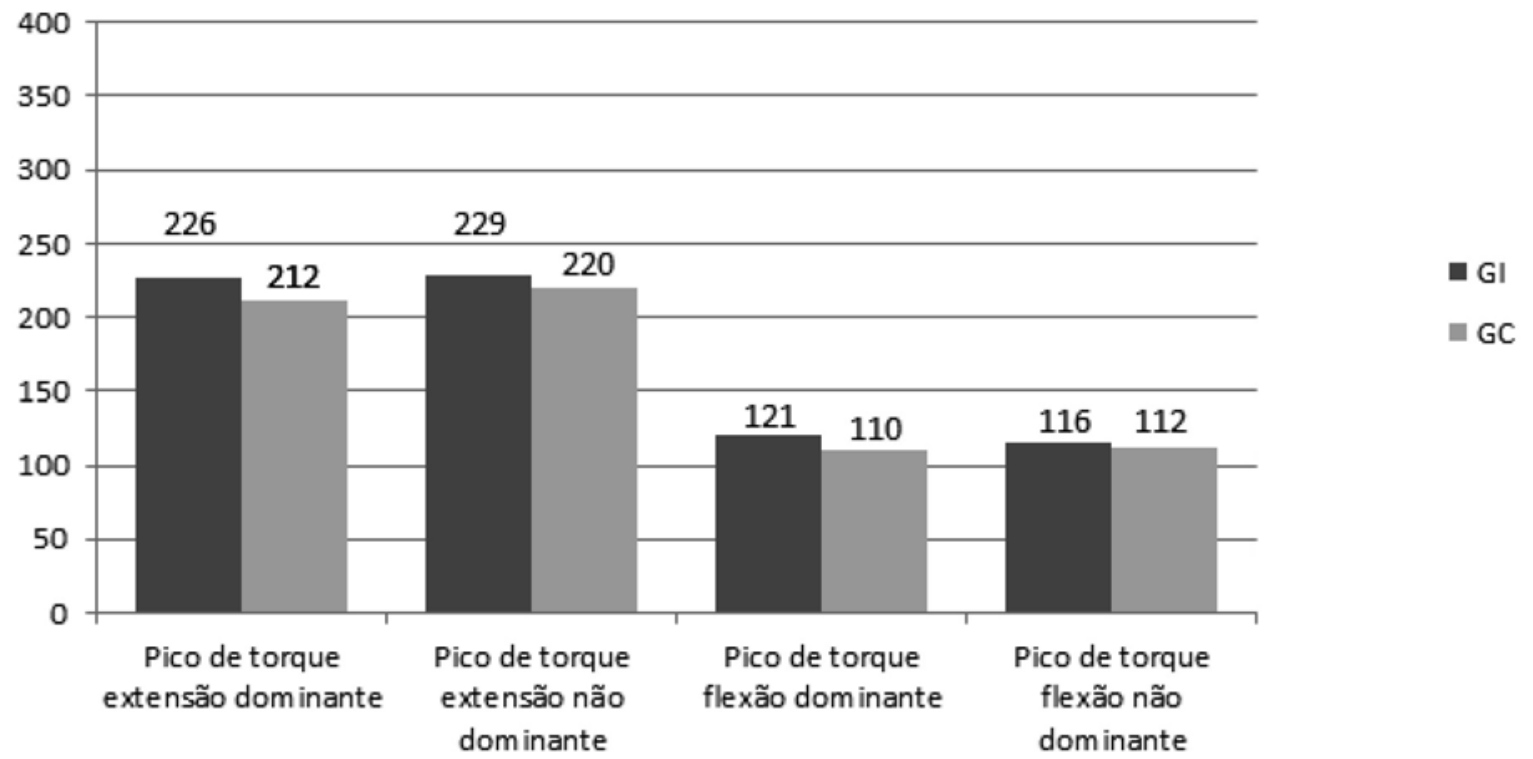

Após analisar os resultados do grupo intervenção e do grupo controle, pré e pós-teste, foi verificado que houve uma piora significativa na variável pico de torque de extensão do membro inferior dominante no grupo que não fez a ALM $(p=0,013)$. Nas demais variáveis, não houve efeitos significativos. Pico de torque extensão membro não dominante $=0,672$; flexão membro dominante $=0,331$; flexão membro não dominante $=0,928$ (dados descritos no gráfico 2 e gráfico 3).

Gráfico 2. Valores de pico de torque do grupo intervenção, pré e pós-teste

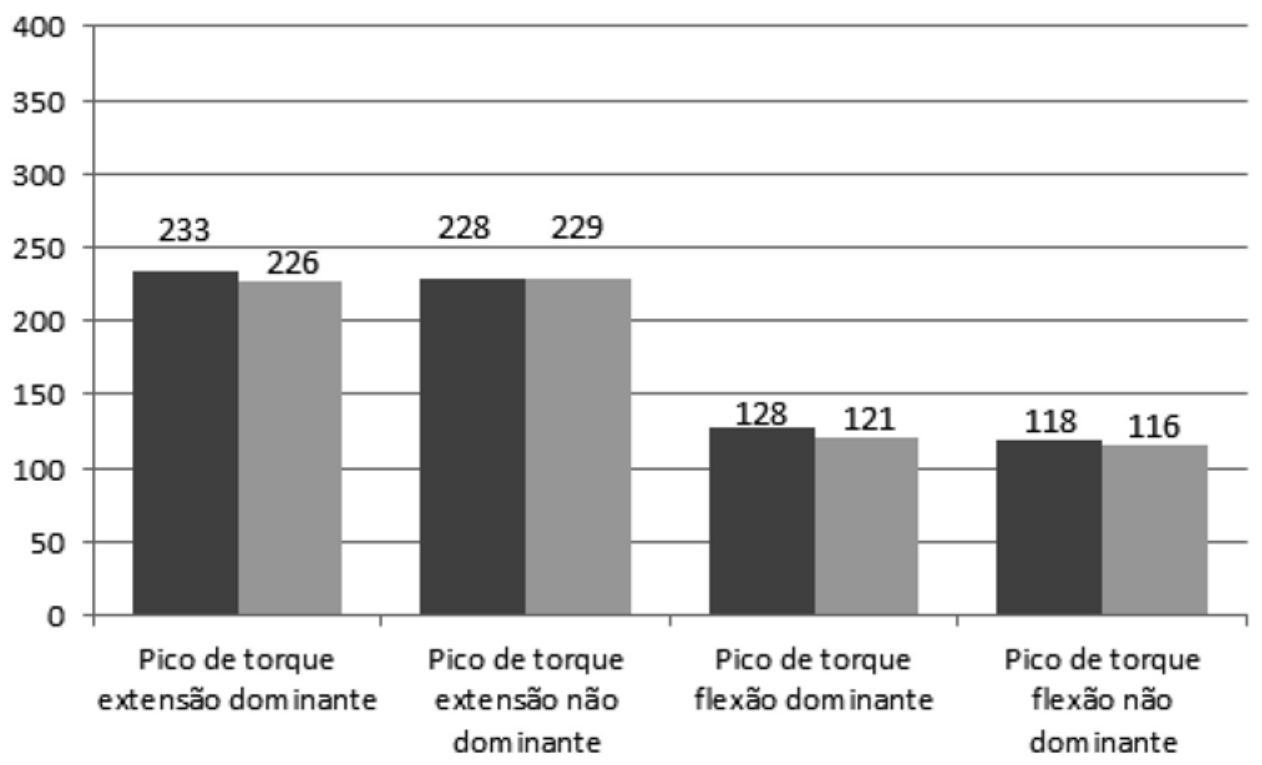




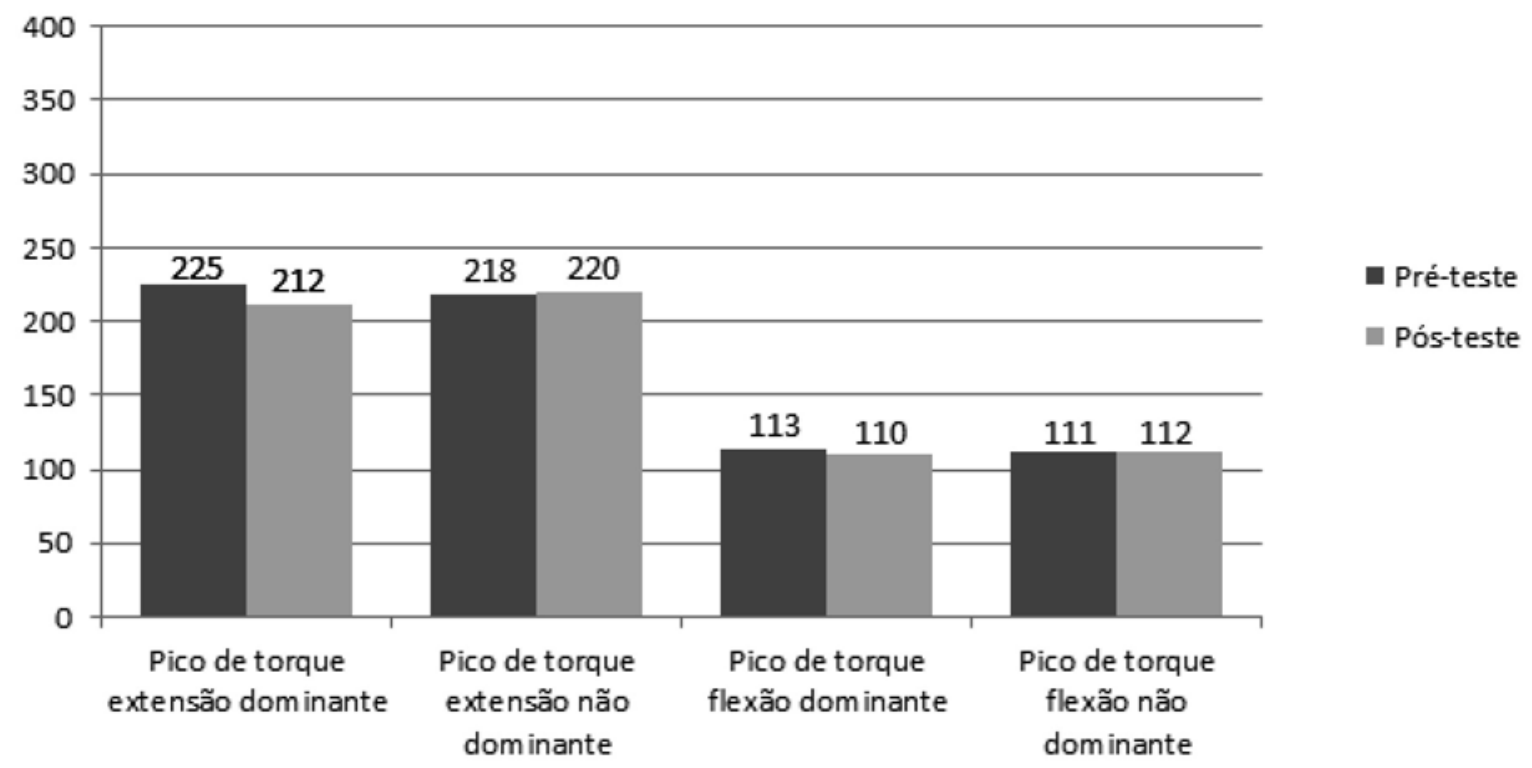

\section{Discussão}

O presente estudo demonstrou que um protocolo de duas semanas de ALM não apresentou efeitos significativos sobre a FM e que o grupo que não fez a ALM apresentou uma piora significativa no pico de torque de extensão do membro dominante. Apesar de esta pesquisa ter adotado um maior número de intervenções de ALM às atletas participantes, quando comparada a outros estudos, ainda assim, a técnica não apresentou alterações de significância sobre a força muscular, o que condiz com a maioria dos estudos que buscaram analisar esta condição 9,10,17,18.

O presente estudo e o de Su et al., 2016, são os úni$\cos$, identificados durante a pesquisa, que utilizaram o aparelho dinamômetro isocinético para avaliar os efeitos da ALM sobre a FM. Não foi encontrado nenhum outro estudo que tenha buscado verificar os efeitos crônicos da ALM na FM e que tenha levado em consideração a avaliação da FM de acordo a dominância do membro inferior. A literatura sugere como uma abordagem crônica, estudos que realizaram um acompanhamento de no mínimo uma semana ${ }^{15}$.

Corroborando com os achados do presente estudo, MacDonald et al. ${ }^{10}$ conduziram uma pesquisa com 11 atletas recreativos do sexo masculino e verificaram aumento da amplitude de movimento do joelho, sem alterar a FM de quadríceps, avaliada em um teste isométrico de contração voluntária máxima dois e dez minutos pós-intervenção. Su et al. ${ }^{17}$ confirmaram esse resultado após realizar um estudo com 30 voluntários (15 homens e 15 mulheres), e verificar que houve aumento da flexibilidade de quadríceps e isquiotibiais, porém sem apresentar diferenças significativas na FM, avaliada no isocinético. O que pode explicar a não observação de mudanças significativas na FM do presente estudo e dos estudos citados, é o tempo de duração de cada série de ALM. Estudos realizados objetivando avaliar os efeitos da massagem demonstraram que uma massagem de curta duração não é capaz de prejudicar a $\mathrm{FM}^{19,20}$. No entanto, estudos que avaliaram os efeitos da massagem com maior tempo de duração, mostraram diminuição da FM de forma aguda ${ }^{21,22}$, o que indica que um maior tempo de duração de ALM pode levar a uma redução da FM nos primeiros momentos após a aplicação. O tempo de duração para cada série de ALM do presente estudo e dos estudos de Su et al. ${ }^{17}$ e de MacDonald et al. ${ }^{10}$, foi de 30 segundos a 1 minuto.

Contrapondo os resultados do presente estudo, Peacock et al. ${ }^{7}$ conduziram uma pesquisa com 11 voluntários do sexo masculino, em que foi realizado o protocolo de ALM nas regiões de peitoral, torácica, lombar, glúteo, isquiotibiais, quadríceps e panturriIha. Os resultados mostraram que a abordagem foi capaz de melhorar os resultados nos testes de desempenho de potência, avaliada com o teste de salto vertical e horizontal, de FM, avaliada no teste de 1-RM indireto, e ainda nos testes de velocidade e agilidade dos sujeitos avaliados. Não se sabe ao certo por qual razão houve melhora FM. Talvez a realização da ALM 
em outras regiões, como o tronco, possa explicar o aumento de força encontrado, pois é visto que abordagens realizadas no tronco levam a melhora do desempenho tanto nos membros inferiores, como nos membros superiores ${ }^{23}$.

Outro achado do presente estudo foi à piora significativa no pico de torque de extensão do membro inferior dominante no grupo que não fez a ALM (grupo controle). Não se sabe ao certo os motivos pelos quais o grupo que não fez a ALM apresentou uma piora nos resultados. Mas o que pode explicar este achado é um maior volume de treinamento realizado pelas atletas durante o período que sucedeu a primeira avaliação isocinética, visto que as atletas estavam no período de pré-temporada. A pré-temporada é comumente descrita por ser um período que há maior intensidade e volume de treinamento ${ }^{24}$. Apesar do aumento das exigências de treinamento gerar adaptações positivas nos atletas, quando o treinamento excede a capacidade de regeneração individual, pode levar a prejuízos do desempenho dos desportistas ${ }^{25}$. No grupo intervenção, a ALM provavelmente minimizou esses prejuízos, visto que estudos apontam que a técnica possui efeitos positivos na recuperação muscular ${ }^{12,14}$.

As limitações deste estudo incluem a não realização de um cálculo amostral, o baixo número de participantes da pesquisa e a falha na investigação de algumas variáveis que possam ter gerado algum viés nos resultados. Entretanto, a realização de uma abordagem crônica de ALM, a utilização de um instrumento de avaliação padrão ouro e a categorização por dominância do membro inferior são vantagens identificadas neste estudo.

\section{Conclusão}

Com base nos resultados do presente estudo, conclui-se que uma abordagem crônica de ALM não foi capaz de gerar mudanças significativas na FM dos extensores e flexores de joelho. Embora o grupo que não fez a ALM ter apresentado uma significativa piora no pico de torque de extensão do membro dominante, não é possível afirmar que a ALM pode contribuir de alguma forma para reduzir os prejuízos sobre a FM. Este estudo, até onde sabemos, é o primeiro a realizar uma abordagem crônica de ALM sobre a FM. Portanto, mais estudos são necessários para apoiar esses achados.

\section{Contribuição dos autores}

Souza SM participou da concepção, delineamento, busca, coleta de dados, análise estatística e interpretação dos dados da pesquisa e redação do artigo científico. Costa Neto JFP participou da concepção, delineamento, coleta de dados da pesquisa e revisão crítica do trabalho. Santos JET participou do delineamento, análise estatística e interpretação dos dados da pesquisa e revisão crítica do trabalho.

\section{Conflitos de interesses}

Nenhum conflito financeiro, legal ou político envolvendo terceiros (governo, empresas e fundações privadas, etc.) foi declarado para nenhum aspecto do trabalho submetido (incluindo, mas não se limitando a subvenções e financiamentos, participação em conselho consultivo, desenho de estudo, preparação de manuscrito, análise estatística, etc.).

\section{Referências}

1. Bizzini M, Hancock D, Impellizzeri F. Suggestions from the field for return to sports participation following anterior cruciate ligament reconstruction: soccer. J Orthop Sports Phys Ther. 2012;42(4):304-12. doi: 10.2519/jospt.2012.4005

2. Cometti G, Maffiuletti NA, Pousson M, Chatard JC, Maffulli N. Isokinetic strength and anaerobic power of elite, subelite and amateur french soccer players. Int J Sports Med. 2001;22(1):45-51. doi: $10.1055 / \mathrm{s}-2001-11331$

3. Silva Neto M, Simões R, Granjeiro Neto JA, Cardone CP. Avaliação isocinética da força muscular em atletas profissionais de futebol feminino. Rev Bras Med Esporte. 2010;16(1):33-5. doi: $\underline{10.1590 / \text { S1517-86922010000100006 }}$

4. Langevin HM, Huijing PA. Communicating about fascia: history, pitfalls, and recommendations. Int J Ther Massage and Bodywork. 2009;2(4):3-8. doi: 10.3822/ijtmb.v2i4.63

5. Barnes MF. The basic science of myofascial release: morphologic change in connective tissue. J Bodywork Mov Ther. 1997;1(4):231-8. doi: 10.1016/S1360-8592(97)80051-4

6. Markovic G. Acute effects of instrument assisted soft tissue mobilization vs. foam rolling on knee and hip range of motion in soccer players. J Bodywork Mov Ther. 2015;19(4):690-6. doi: 10.1016/j.jbmt.2015.04.0108592(97)80051-4

7. Peacock CA, Krein DD, Silver TA, Sanders GJ, Carlowitz KPAV. An acute bout of self-myofascial release in the form of foam rolling improves performance testing. Int J Exerc Sci. 2014;7(3):202-11.

8. Phillips J, Diggin D, King DL, Sforzo GA. Effect of varying self-myofascial release duration on subsequent athletic performance. J Strength Cond Res. 2018;50:801. doi: 10.1519/ JSC.0000000000002751 
9. Healey KC, Hatfield DL, Blanpied P, Dorfman LR, Riebe $D$. The effects of myofascial release with foam rolling on performance. J Strength Cond Res. 2014;28(1):61-8. doi: 10.1519/ ISC.0b013e3182956569

10. MacDonald GZ, Penney MD, Mullaley ME, Cuconato AL, Drake $C D$, Behm DG et al. An acute bout of self-myofascial release increases range of motion without a subsequent decrease in muscle activation or force. J Strength Cond Res. 2013;27(3):812-21. doi: $10.1519 /$ JSC.0b013e31825c2bc1

11. Fleckenstein J, Wilke J, Vogt L, Banzer W. Preventive and regenerative foam rolling are equally effective in reducing fatiguerelated impairments of muscle function following exercise. J Sports Sci Med. 2017;16(4):474-79.

12. MacDonald GZ, Button DC, Drinkwater EJ, Behm DG. Foam rolling as a recovery tool after an intense bout of physical activity. Med Sci Sports Exerc. 2014;46(1):131-42. doi: 10.1249/ MSS.0b013e3182a123db

13. Pearcey GEP, Bradbury-Squires DJ, Kawamoto JE, Drinkwater EJ, Behm DG, Button DC. Foam rolling for delayed-onset muscle soreness and recovery of dynamic performance measures. J Athl Train. 2015;50(1):5-13. doi: 10.4085/1062-6050-50.1.01

14. Wiewelhove T, Döweling A, Schneider C, Hottenrott L, Meyer $\mathrm{T}$, Kellmann $\mathrm{M}$ et al. A meta-analysis of the effects of foam rolling on performance and recovery. Front Physiol. 2019;10:376. doi: 10.3389/fphys.2019.00376

15. Beardsley C, Skarabot J. Effects of self-myofascial release: A systematic review. J Bodywork Mov Ther. 2015;19(4):747-58. doi: 10.1016/j.jbmt.2015.08.007

16. Grabow L, Young JD, Alcock LR, Quigley PJ, Byrne JM, Granacher $U$ et al. Higher quadriceps roller massage forces do not amplify range-of-motion increases or impair strength and jump performance. J Strength Cond Res. 2017;32(11):1. doi: 10.1519/ ISC.0000000000001906

17. Su H, Chang NJ, Wu WL, Guo LY, Chu IH. Acute effects of foam rolling, static stretching, and dynamic stretching during warmups on muscular flexibility and strength in young adults. J Sport Rehabil. 2016;26(6):469-477. doi: 10.1123/jsr.2016-0102

18. Sullivan KM, Silvey DB, Button DC, Behm DG. Roller-massager application to the hamstrings increases sit-and-reach range of motion within five to ten seconds without performance impairments. Int J Sports Phys Ther. 2013;8(3):228-36.

19. Silva LPO, Oliveira MFM, Caputo F. Métodos de recuperação pós-exercício. Rev. Educ. Fis/UEM. 2013;24(3):489-508. doi: 10.4025/reveducfis.v24.3.17487

20. McKechnie GJB, Young WB, Behm DG. Acute effects of two massage techniques on ankle joint flexibility and power of the plantar flexors. J Sports Sci Med. 2007;6(4):498-504.
21. Arroyo-Morales M, Olea N, Martínez MM, Hidalgo-Lozano A, Ruiz-Rodríguez C, Díaz-Rodríguez L. Psychophysiological effects of massage-myofascial release after exercise: A randomized shamcontrol study. J Altern Complement Med. 2008;14(10):1223-9. doi: 10.1089/acm.2008.0253

22. Wiktorsson-Moller M, Oberg B, Ekstrand J, Gillquist J. Effects of warming up, massage, and stretching on range of motion and muscle strength in the lower extremity. Am J Sports Med. 1983; 11(4):249-52. doi: $10.1177 / 036354658301100412$

23. Kim Y, Kim E, Gong W. The effects of trunk stability exercise using PNF on the functional reach test and muscle activities of stroke patients. J Phys Ther Sci. 2011;23(5):699-702. doi: 10.1589/ jpts.23.699

24. Owen AL, Forsyth JJ, Wong DP, Dellal A, Connelly SP, Chamari K. Heart rate-based training intensity and its impact on injury incidence among elite-level professional soccer players. J Strength Cond Res. 2015;29(6):1705-12. doi: 10.1519/ JSC.0000000000000810

25. Matos FO, Samulski DM, Lima JRP, Prado LS. Cargas elevadas de treinamento alteram funções cognitivas em jogadores de futebol. Rev Bras Med Esporte. 2014;20(5):388-393. doi: 10.1590/1517-86922014200501274 